\title{
Collimation of a spherical collisionless particles stream in Kerr space-time
}

\author{
Kentaro Takamit and Yasufumi Kojimat \\ Department of Physics, Hiroshima University, Higashi-Hiroshima 739-8526, Japan
}

\begin{abstract}
We examine the propagation of collisionless particles emitted from a spherical shell to infinity. The number distribution at infinity, calculated as a function of the polar angle, exhibits a small deviation from uniformity. The number of particles moving from the polar region toward the equatorial plane is slightly larger than that of particles in the opposite direction, for an emission radius $>4.5 \mathrm{M}$ in extreme Kerr space-time. This means that the black hole spin exerts an anti-collimation effect on the particles stream propagating along the rotation axis. We also confirm this property in the weak field limit. The quadrupole moment of the central object produces a force toward the equatorial plane. For a smaller emission radius $r<4.5 M$, the absorption of particles into the black hole, the non-uniformity and/or the anisotropy of the emission distribution become much more important.

PACS numbers: 04.20.Cv, 04.70.-s, 95.30.Sf, 97.60.Lf, 98.38.Fs, 98.58.Fd
\end{abstract}

\section{Introduction}

Astrophysical relativistic jets are commonly observed in AGNs, microquasars and gamma ray bursts. The Lorentz factor of the outflow motion is $\gamma \approx 10$ in AGNs, $\gamma \approx 2-5$ for microquasars and $\gamma \approx 100$ for gamma ray bursts. It is considered likely that a central black hole is related to the formation of such jets due to the large luminosity and short timescale of the jets. However, the actual mechanism of such collimated outflows has not yet been resolved, although many theories have been proposed. One possible mechanism that has been suggested relates to magnetic hoop stress. Recently, large-scale MHD simulations of jets have been successfully performed by several authors. For example, McKinney [1] numerically studied the hydrodynamical flow emitted from an accretion disk, where the jet formation extends to a distance of ten thousand times the radius of the central black hole. In order for a collimated structure to form, the magnetic pressure has to be sufficiently large in comparison to the ambient pressure of the surrounding medium.

In this paper, we explore an alternative possibility. That is, we investigate a mechanism involving the black hole spin itself. The strong gravitational field near a black

$\dagger$ Email: takami@theo.phys.sci.hiroshima-u.ac.jp

‡ Email: kojima@theo.phys.sci.hiroshima-u.ac.jp 
hole also affects the flow of particles(e.g., [2, 3]). The effect of gravity may be important when the emission region is concentrated in the vicinity of the central black hole. Bičák et al. 4] discussed this effect by calculating the geodesics of some particles in Kerr spacetime. de Felice and Carlotto [5] took into account the slow loss of angular momentum and energy of the particles moving along the geodesics. They derived a certain condition for such dissipative collimation and discussed its astrophysical relevance [6, 7]. Semerák [8] considered the effect of a spinning particle in Kerr space-time. More recently, Gariel et al. [9] discussed the collimation of geodesics by using cylindrical coordinates. These authors pointed out the possibility of collimated outflow occurring along the rotation axis of a Kerr black hole. The axis is a unique preferable direction of the flow, and some trajectories are in fact concentrated along the axis. Despite the fact that the possible collimation mechanisms in Kerr space-time have been discussed extensively, it is not clear what fraction of the flow (the ratio of the flow along the rotation axis to the overall emission) is collimated. For example, if particle emissions are selected with orbital parameters suitable for the flow along the axis, then the collimated flow is realized. It is therefore important to evaluate the degree of the collimation and the results will be useful for the astrophysical application.

The problem is closely related to the emission process itself. To tackle this problem, we present a model for studying geometrical collimation. In our model, billions of particles are isotropically emitted from a shell with a constant radius covering a central black hole, and the trajectories are numerically calculated. We use a test-particle (dust) approximation to calculate each trajectory. The collision of particles is ignored in order to evaluate the geometrical effect in an idealized situation. The collisional hydrodynamics may work toward local isotropy, and may partially hide the effect. The angular distribution of the particles at the emission radius is assumed to be uniform, but at infinity some deviation from a uniform distribution is produced by Kerr black holes. In this way, we study to what extent a rotating black hole affects the collimation of collisionless particles. Our model is not rigorously realistic, but is instead an idealized mathematical model which is useful in evaluating what fraction of the flow is collimated.

The organization of this paper is as follows. In Section 2, we summarize our models. The numerical distribution of the trajectories of the particles at infinity is calculated as a function of the polar angle, the particles having been emitted with a uniform distribution from a 'spherical' shell. It is not easy to define a spherically symmetric surface in non-spherical space-times. Two dimensional spatial surface with a constant radius is approximately regarded as a sphere. This definition, which is valid for asymptotically large radius, is used in this paper. The numerical results in Boyer-Lindquist coordinate are given in Section 3. In order to consider the physical mechanism, the same calculation is performed in weak gravity. Asymptotically Cartesian and mass centered to order $\mathrm{N}$ (ACMC-N) coordinate is used and the results are given in Section 4. As the emission radius becomes smaller, a larger number of particles which are emitted outwardly are absorbed into the central black hole. In this case, the spatial uniformity and the isotropy of the particle distribution on the emission sphere become meaningless. In order to 
Collimation of a spherical collisionless particles stream

demonstrate the absorption effect, some results are also shown in Section 5. Finally, a discussion of our results is given in Section 6. We use units of $c=G=1$ in this paper.

\section{Model and method}

The Kerr space-time for a black hole with mass $M$ and angular momentum $a M$ is described in the Boyer-Lindquist coordinates $(t, r, \theta, \phi)$ as

$$
d s^{2}=-e^{2 \nu} d t^{2}+e^{2 \psi}(d \phi-\omega d t)^{2}+\frac{\rho^{2}}{\Delta} d r^{2}+\rho^{2} d \theta^{2}
$$

where

$$
\begin{aligned}
& e^{2 \nu}=\frac{\Delta \rho^{2}}{A}, e^{2 \psi}=\frac{A \sin ^{2} \theta}{\rho^{2}}, \omega=\frac{2 a M r}{A}, \\
& \rho^{2}=r^{2}+a^{2} \cos ^{2} \theta, \Delta=r^{2}+a^{2}-2 M r, A=\left(r^{2}+a^{2}\right)^{2}-a^{2} \Delta \sin ^{2} \theta .
\end{aligned}
$$

There are three constants related to the geodesic motion in Kerr space-time: the energy $E$, the $z$ component of the angular momentum $L_{z}$ and Carter's constant $\mathcal{Q}$ [10, 11, 12]. The 4-momentum $p_{\mu}$ of a particle with a rest mass $m$ is therefore written as

$$
p_{\mu}=\left(-E, \pm \frac{R^{1 / 2}}{\Delta}, \pm \Theta^{1 / 2}, L_{z}\right) \text {, }
$$

where

$$
\begin{aligned}
& R=\left(E\left(r^{2}+a^{2}\right)-L_{z} a\right)^{2}-\Delta\left[m^{2} r^{2}+\left(L_{z}-a E\right)^{2}+\mathcal{Q}\right], \\
& \Theta=\mathcal{Q}-\cos ^{2} \theta\left[a^{2}\left(m^{2}-E^{2}\right)+\frac{L_{z}^{2}}{\sin ^{2} \theta}\right] .
\end{aligned}
$$

In order to allow for the emission model of particles to be considered, we introduce a reference frame $(\hat{t}, \hat{r}, \hat{\theta}, \hat{\phi})$ measured by zero-angular momentum observers (ZAMO) (e.g.,[12]). The reference frame is represented by four orthogonal unit vectors, i.e., one timelike vector $\mathbf{e}_{(0)}$ and three spacelike vectors $\mathbf{e}_{(n)}(n=1,2,3)$. The explicit components

are given by $e_{(0)}^{\mu}=\left[e^{-\nu}, 0,0, \omega e^{-\nu}\right], e_{(1)}^{\mu}=\left[0, \Delta^{1 / 2} / \rho, 0,0\right], e_{(2)}^{\mu}=[0,0,1 / \rho, 0]$ and $e_{(3)}^{\mu}=\left[0,0,0, e^{-\psi}\right]$. The 4-momentum $\mathbf{p}$ of a particle is expressed by

$$
\mathbf{p}=\hat{E} \mathbf{e}_{(0)}+\hat{p}\left(\cos \hat{\theta} \mathbf{e}_{(1)}+\sin \hat{\theta} \cos \hat{\phi} \mathbf{e}_{(2)}+\sin \hat{\theta} \sin \hat{\phi} \mathbf{e}_{(3)}\right),
$$

where the angles $\hat{\theta}$ and $\hat{\phi}$ are determined by the emission direction of the particle. The energy and the linear momentum satisfy the special relativistic relation $\hat{p}=\left(\hat{E}^{2}-m^{2}\right)^{1 / 2}$, $\hat{E}=m \hat{\gamma}$, where $\hat{\gamma}=\left(1-\hat{v}^{2}\right)^{-1 / 2}$ and $\hat{v}$ is the velocity of the test particle.

From Eqs.(3) and (6), the constants of motion $E, L_{z}, \mathcal{Q}$ can be expressed by quantities measured by a ZAMO,

$$
\begin{aligned}
& E=\hat{E} e^{\nu}+\omega L_{z}, \\
& L_{z}=\hat{p} \sin \hat{\theta} \sin \hat{\phi} e^{\psi} \\
& \mathcal{Q}=(\hat{p} \rho \sin \hat{\theta} \cos \hat{\phi})^{2}+\cos ^{2} \theta\left[a^{2}\left(m^{2}-E^{2}\right)+\frac{L_{z}^{2}}{\sin ^{2} \theta}\right] .
\end{aligned}
$$


We consider a particle moving along the geodesic curve without any other forces, so that $r-\theta$ motion can be calculated by integrating the differential equation

$$
\frac{d \theta}{d r}= \pm \sqrt{\frac{\Theta}{R}}
$$

with initial condition $(r, \theta)=\left(r_{\mathrm{e}}, \theta_{\mathrm{e}}\right)$. The numerical integration method is a fourthorder Runge-Kutta method with an adaptive step size, and the numerical errors are adjusted to be small enough. In order to check the validity of our numerical integration, we calculated the same problem as Bičák et al. 4] and obtained the same results.

We consider the particle distribution, $f(\cos \theta)$, at infinity $r_{\infty}$, when a large number of test particles, $N_{\text {tot }}$, are emitted from a shell of radius $r_{\mathrm{e}}$. The particle distribution at $r_{\mathrm{e}}$ is assumed to be uniform, that is, the number of particles $d N$ emitted from $\theta_{\mathrm{e}}$ to $\theta_{\mathrm{e}}+d \theta_{\mathrm{e}}$ is $d N=\left(N_{\mathrm{tot}} / 4 \pi\right)\left(2 \pi \sin \theta_{\mathrm{e}} d \theta_{\mathrm{e}}\right)$ for $0 \leqq \theta_{\mathrm{e}} \leqq \pi$. As for the emission model at $\left(r_{\mathrm{e}}, \theta_{\mathrm{e}}\right)$, we also assume that the particles have the same energy $\hat{E}$, and that the emission direction is isotropic with respect to the angles $\hat{\theta}$ and $\hat{\phi}$. The angle $\hat{\theta}$ might be limited to $0 \leqq \hat{\theta} \leqq \pi / 2$, since most of the particles satisfying the emission angle $\pi / 2 \leqq \hat{\theta} \leqq \pi$ are absorbed by the black hole and can therefore be ignored from the beginning. In the numerical calculation, the directions $\hat{\theta}$ and $\hat{\phi}$ are randomly chosen at $r_{\mathrm{e}}$, and the constants of motion can be calculated by Eqs.(17)-(9). The asymptotic value $\theta$ of each particle is numerically evaluated by solving Eq.(10) to $r_{\infty}$, where $r_{\infty}$ is a large number satisfying $r_{\infty} \gg M$, which is set to 'infinity' in the numerical calculation. By calculating $N_{\text {tot }}$ trajectories, we obtain the number distribution $f_{*}(\cos \theta)$.

In order to study the geometrical effects, we are not interested in a fine-grained distribution $f_{*}$ but in a coarse-grained distribution, i.e., an averaged distribution on a larger angular scale. In the numerical calculations, we divide $\cos \theta(0 \leqq \cos \theta \leqq 1)$ into $N_{0}$ segments with a small interval $d \cos \theta$, and count the number of the trajectories which arrive at the region between $\cos \theta$ and $\cos \theta+d \cos \theta$. The number is approximated by $f(\cos \theta) d \cos \theta$, and the function $f(\cos \theta)$ is numerically constructed.

In our model, the isotropy of the emission direction and the spatial uniformity are assigned by a ZAMO at a constant radius in Boyer-Lindquist coordinates. If the emission is uniform with respect to the proper surface element at $r_{\mathrm{e}}$, then the number of particles $d N$ should be modified as $d N=g\left(N_{\text {tot }} / 4 \pi\right)\left(2 \pi \sin \theta_{\mathrm{e}} d \theta_{\mathrm{e}}\right)$ for $0 \leqq \theta_{\mathrm{e}} \leqq \pi$. The correction factor $g$ is estimated as $g=2 A^{1 / 2} /\left(\int_{0}^{\pi} A^{1 / 2} \sin \theta d \theta\right) \approx 1+a^{2}\left(r_{\mathrm{e}}-2 M\right)\left(3 \cos ^{2} \theta-1\right) /\left(6 r_{\mathrm{e}}^{3}\right)$, which gives a small variation of $5 \times 10^{-3}$ for $r_{\mathrm{e}}=4.5 \mathrm{M}$. The correction $|g-1|$ seems to increase as $r_{e}$ decreases. We have numerically evaluated the factor and found that there is a maximum value at a certain radius $r_{e}$. For example, in extreme Kerr space-time $a=M$, we found that $|g-1|<1.5 \times 10^{-2}$, where the maximum occurs at $r_{e} \sim 2.4 M$ and $\theta=0, \pi$. We actually include this correction factor into the numerical calculations in Section 3 and 5, and the obtained results are not significantly different, since other physical effects are much larger in the magnitude. 

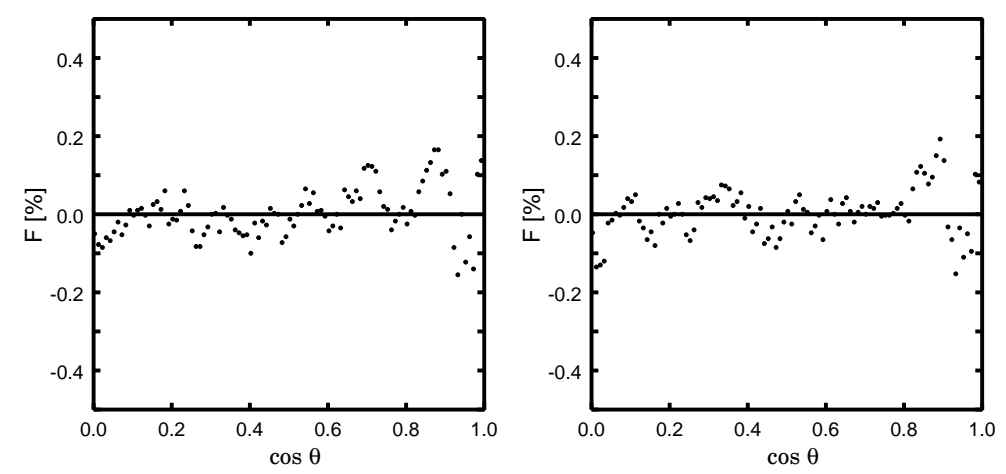

Figure 1. Deviation from uniform distribution as a function of $\cos \theta$. The left panel shows the results obtained for a flat space-time, and the right panel shows the results obtained for a Schwarzschild space-time.

\section{Effect of geometry on propagation}

In principle, the number distribution function $f(\cos \theta)$ ranges from 0 to $N_{\text {tot }}$ and clearly depends on $N_{\text {tot }}$. Therefore, this property is not suitable when comparing, for example, numerical results obtained using different total numbers of particles. The deviation from uniform distribution is more important. We therefore use the following normalized distribution $F(\cos \theta)$ defined by

$$
F(\cos \theta)=\frac{f(\cos \theta)-\langle f\rangle}{\langle f\rangle} \times 100[\%],
$$

where

$$
\langle f\rangle=\frac{\int_{0}^{1} f(\cos \theta) d(\cos \theta)}{\int_{0}^{1} d(\cos \theta)} .
$$

This normalized function becomes $F=0$ when $f$ does not depend on $\cos \theta$, i.e., in the case of a spherically symmetrical distribution.

First, we present the two test problems. The distributions are calculated for a flat space-time and a Schwarzschild space-time. We expect $F=0$, for $0 \leqq \cos \theta \leqq 1$ since both space-times have spherical symmetry. We adopt $N_{\text {tot }}=2 \times 10^{9}, r_{\mathrm{e}}=4.5 \mathrm{M}$, $r_{\infty}=500 M$ and $\hat{v}=0.9(\hat{\gamma} \sim 2)$ in the numerical calculations. The results are shown in Figure 1. There are small irregular deviations from 0 in the number distributions $F$. These small deviations are $\sim 0.2 \%$ at most, and arise from the finite numbers $N_{\text {tot }}$ and $N_{0}$. In constructing $F$, we divide the angle into $N_{0}(=100)$ segments. If $N_{\text {tot }}\left(=2 \times 10^{9}\right)$ trajectories are randomly distributed, then the mean value is $N_{\text {tot }} / N_{0}$, and the deviation from the average is $\left(N_{0} / N_{\text {tot }}\right)^{1 / 2} \approx 2 \times 10^{-4}$. Additionally, randomly chosen emission angles, $\hat{\theta}$ and $\hat{\phi}$, are involved in the numerical calculation, so that the deviation increases by a certain factor. We numerically checked that the statistical error is roughly proportional to $N_{\text {tot }}^{-1 / 2}$. The error therefore becomes 0 in the limit of $N_{\text {tot }} \rightarrow \infty$. Fortunately, this 'noise' level is sufficiently low to allow the effect of black hole spin to be examined, as discussed below. 


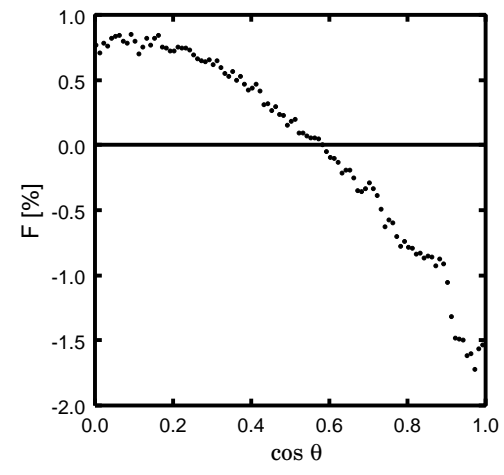

Figure 2. The same as Figure 1, but for the extreme Kerr space-time.

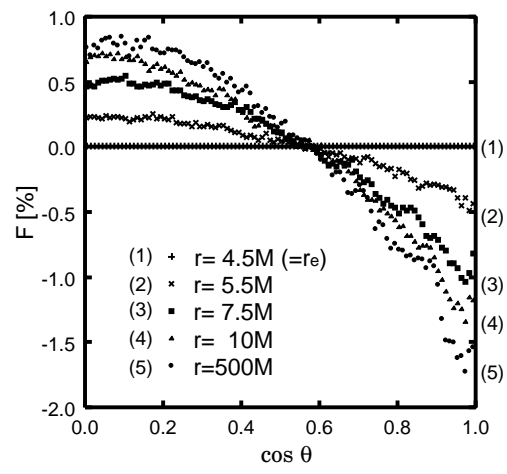

Figure 3. Evolution of normalized number distribution for different values of the radial coordinate $r$.

Next, we calculate the number distribution $F$ for the extreme Kerr space-time. We adopt the same parameters as those used in the test calculations. The results are shown in Figure 2. The number distribution $F$ exhibits a larger deviation, where there is a deficit of $F \sim-1.7 \%$ near the polar regions and an excess of $F \sim 0.8 \%$ near the equatorial region. These values are obviously larger than the statistical error of $\sim 0.2 \%$ in test calculations. The results, however, are opposite to our expectation that the spin of the black hole would cause the particles to be collimated along the rotation axis.

Figure 3 shows how the number distribution $F$ changes with the propagation distance. The distributions for some selected radii are calculated from an initially uniform distribution. It can easily be seen that the deviation from the average gradually increases with $r$ from the emitting radius $r_{\mathrm{e}}=4.5 \mathrm{M}$ to $r_{\infty}=500 \mathrm{M}$. This increase almost stops for $r>10 M$, where the effect of the Kerr space-time becomes very small $(a / r)^{2} \sim 10^{-2}$. This result indicates that the particles tend to fall toward the equatorial plane due to the Kerr black hole. This feature has been partially discussed by Bičák et al. 4.

In order to express the above geometrical deviation in a simple way, we calculate the function $F$ for various values of the Kerr parameter $a$, the emitting radius $r_{\mathrm{e}}$ and the emitting velocity $\hat{\gamma}$. It is found that the following empirical relation is well fitted to the numerical results of approximately 20 models in the ranges $0 \leq a \leq M$, $4.5 M \leq r_{\mathrm{e}} \leq 10 M$ and $2 \leq \hat{\gamma} \leq 10^{3}$,

$$
F(\cos \theta) \sim-9.2 \times 10\left(\frac{a}{M}\right)^{2}\left(\frac{r_{\mathrm{e}}}{M}\right)^{-2.5}\left(1+\frac{1}{\hat{\gamma}^{2}}\right)\left(\cos ^{2} \theta-\frac{1}{3}\right),
$$

where the angular part is the Legendre polynomial of $l=2$. From this relation, the most important factor affecting the geometrical deviation is the emission radius $r_{\mathrm{e}}$. Our calculation in this section is limited to $r_{\mathrm{e}} \geq 4.5 \mathrm{M}$, but the deviation can be increased further for particles emitted in the vicinity of the black hole. As shown in Section 5. however, the extrapolation of Eq.(13) for smaller values of $r_{\mathrm{e}}$ is not particularly meaningful, since the fraction of absorbed trajectories becomes more important. 


\section{Weak gravity with quadrupole moment}

In order to examine the deviation from spherical symmetry, we consider the weak gravity limit of Kerr space-time. We introduce asymptotically Cartesian and mass centered to order $\mathrm{N}(\mathrm{ACMC}-\mathrm{N})$ coordinate $\operatorname{system}\left(t^{\prime}, r^{\prime}, \theta^{\prime}, \phi^{\prime}\right)$ [13]. In the ACMC-2 coordinate system, which is the lowest order containing non-spherical terms, the Kerr metric is written as follows:

$$
\begin{aligned}
& g_{0^{\prime} 0^{\prime}}=-1+\frac{2 M}{r^{\prime}}-\frac{3 M a^{2} \cos ^{2} \theta^{\prime}}{r^{\prime 3}}, \\
& g_{0^{\prime} 3^{\prime}}=r^{\prime} \sin \theta^{\prime}\left(-\frac{2 M a \sin \theta^{\prime}}{r^{\prime 2}}+\frac{5 M a^{3} \sin \theta^{\prime} \cos ^{2} \theta^{\prime}}{r^{\prime 4}}\right), \\
& g_{1^{\prime} 1^{\prime}}=1+\frac{2 M}{r^{\prime}}+\frac{4 M^{2}-a^{2}}{r^{\prime 2}}+\frac{8 M^{3}-4 M a^{2}-M a^{2} \cos ^{2} \theta^{\prime}}{r^{\prime 3}}, \\
& g_{2^{\prime} 2^{\prime}}=r^{\prime 2}\left(1+\frac{a^{2}}{r^{\prime 2}}\right), \\
& g_{3^{\prime} 3^{\prime}}=r^{\prime 2} \sin ^{2} \theta^{\prime}\left(1+\frac{a^{2}}{r^{\prime 2}}+\frac{2 M a^{2} \sin ^{2} \theta^{\prime}}{r^{\prime 3}}\right), \\
& g_{1^{\prime} 2^{\prime}}=r^{\prime}\left(-\frac{2 M a^{2} \cos \theta^{\prime} \sin \theta^{\prime}}{r^{\prime 3}}\right),
\end{aligned}
$$

where the relations to the Boyer-Lindquist coordinates $(t, r, \theta, \phi)$ are

$$
\begin{aligned}
& r=r^{\prime}+\frac{a^{2} \cos ^{2} \theta^{\prime}}{2 r^{\prime}}, \quad \theta=\theta^{\prime}-\frac{a^{2} \cos \theta^{\prime} \sin \theta^{\prime}}{2 r^{\prime 2}}, \\
& \phi=\phi^{\prime}, \quad t=t^{\prime} .
\end{aligned}
$$

One of the important non-spherical effects is the quadrupole field in the Newtonian level, $g_{0^{\prime} 0^{\prime}}=-\left(1+2 \Phi_{N}\right)$, where

$$
\Phi_{N}=-\frac{M}{r^{\prime}}+\frac{3 M a^{2} \cos ^{2} \theta^{\prime}}{2 r^{\prime 3}} .
$$

A particle in this potential field receives an acceleration in the $\theta^{\prime}$-direction as

$$
\alpha_{\theta^{\prime}}=\frac{3 M a^{2} \sin 2 \theta^{\prime}}{2 r^{\prime 4}}
$$

This is positive in the upper half plane $\left(0<\theta^{\prime}<\pi / 2\right)$, while negative for $\pi / 2<\theta^{\prime}<\pi$. The quadrupole field therefore bends the orbit of a particle toward the direction of equatorial plane. The quadrupole feature of gravity should be one of the mechanisms for the anti-collimation shown in Section 3. In the following, we examine numerically the magnitude of the deviation in the ensemble of particles which is caused by the quadrupole moment. We calculate the particle distribution $F(\cos \theta)$ using Eqs.(14)(19) and geodesic equations, in the same way as done in Section 3. In this space-time, we solve the second-order differential equation for the geodesic without using Carter's constant $\mathcal{Q}$. The results for $a=M, r_{\mathrm{e}}^{\prime}=4.5 M$ and $\hat{v}=0.9(\hat{\gamma} \sim 2)$ are shown in Figure 4. The number distribution $F$ has a deficit near the polar region and an excess near the equatorial region. This behavior is the same as Figure 2. The concentration of 


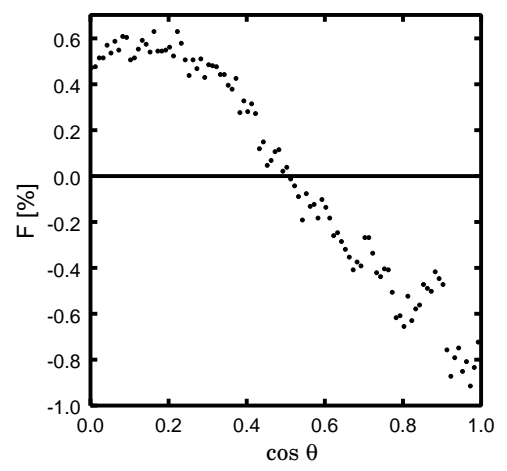

Figure 4. Deviation from uniform distribution at infinity for $a=M$ in the ACMC-2 coordinate, $r_{\mathrm{e}}^{\prime}=4.5 M$ and $\hat{v}=0.9(\hat{\gamma} \sim 2)$.

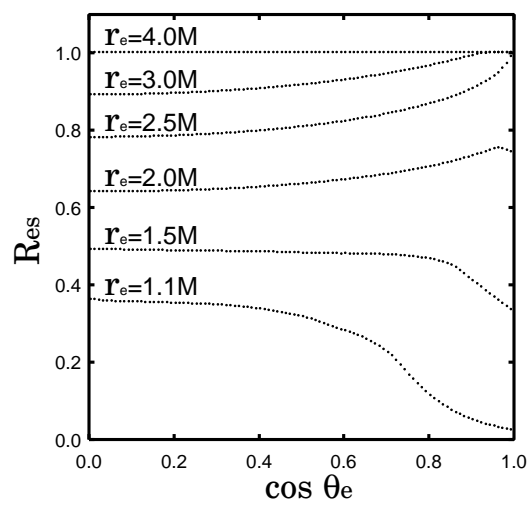

Figure 5. Escape fraction for various emission radii $r_{\mathrm{e}}$ in the extreme Kerr spacetime. The escape fraction is the ratio of the number of particles which have escaped to infinity to the total number of particles emitted from $\left(r_{\mathrm{e}}, \theta_{\mathrm{e}}\right)$.

particles toward the equatorial plane can partially be explained by the mass quadrupole moment of the central object. The deviation of Figure 2 is roughly twice as large as that of Figure 4. It is impossible to exactly compare results in different spacetimes exactly. The emission models are different. For example, the constant radius $r_{\mathrm{e}}^{\prime}=4.5 M$ in ACMC-2 coordinate does not agree with the radius $r_{\mathrm{e}}=4.5 M$ in the Boyer-Lindquist coordinate. The correspondence is approximately valid. There is a common feature of particle concentration toward the equatorial plane, although exact comparison between two different coordinates is impossible, and hence there is still a problem in the magnitude. In the weak field limit, the feature is explained by a mass quadrupole term. The feature still appears in much strong field, as calculated in Section 3.

\section{Absorption effect}

The number of orbits in which particles are absorbed into the black hole increases as the distance between the emitting surface and the black hole decreases. Figure 5 shows the escape fraction $R_{\mathrm{e} s}$ as a function of $\cos \theta_{\mathrm{e}}$ in the extreme Kerr space-time. The 

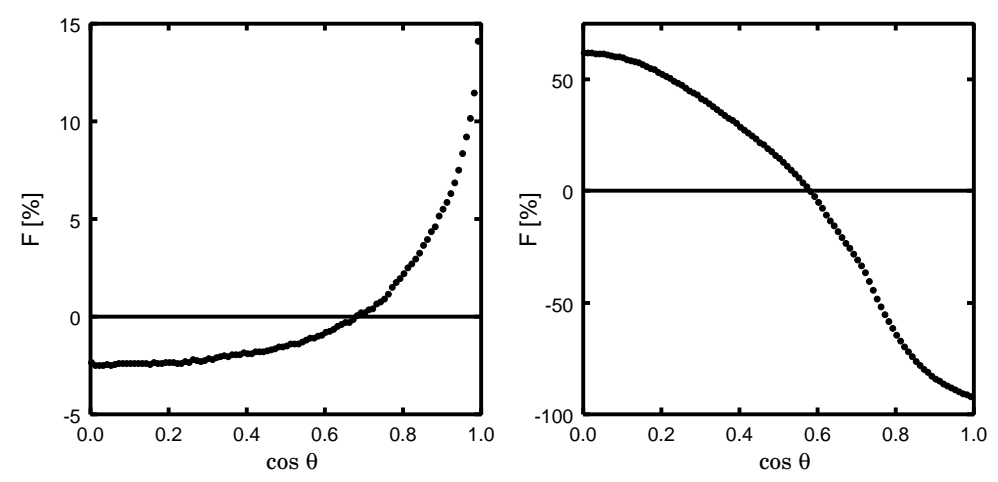

Figure 6. Deviation from uniform distribution at infinity. The left panel shows the results for emission radius $r_{\mathrm{e}}=2.5 \mathrm{M}$, and the right for $r_{\mathrm{e}}=1.1 \mathrm{M}$.

escape fraction is the ratio of the number of escaped particles to the total number of particles emitted from $\left(r_{\mathrm{e}}, \theta_{\mathrm{e}}\right)$. The initial velocity of the particles is chosen as $\hat{v}=0.99999(\hat{\gamma} \sim 224)$. For a large emission radius, the escape fraction is almost unity, which indicates that in-falling orbits to the black hole can be ignored. As the radius $r_{\mathrm{e}}$ decreases, the escape fraction significantly decreases. Figure 5 shows that the distribution of particles escaping to infinity or being absorbed into the black hole is not uniform, showing a slight dependence on the angle $\theta_{\mathrm{e}}$. Such dependence indicates that the emission distribution can be regarded as being non-uniform. This property is important since the non-uniformity at the emission surface naturally leads to the nonuniform outflow at infinity. The trajectories of particles emitted at an angle $\theta_{\mathrm{e}}$ do not necessarily have the same angle $\theta=\theta_{\mathrm{e}}$ at infinity, as the correspondence between the polar angles is somewhat different at the time of emission and at the time of observation due to its modification during the propagation of the particles. It is seldom the case that a non-uniform distribution becomes a uniform distribution at infinity, and therefore the existence of non-uniformity at emission might be important.

The results shown in Figure [6] illustrate the effect of the orbits in which particles are absorbed in the extreme Kerr black hole. The left and right panels show the results obtained for an emission radius $r_{\mathrm{e}}=2.5 M$ and $r_{\mathrm{e}}=1.1 M$, respectively. The initial velocity is $\hat{v}=0.99999(\hat{\gamma} \sim 224)$, and the other parameters are the same as those used in the test calculations. Regarding emission for $r_{\mathrm{e}}=2.5 \mathrm{M}$, there is an excess of $F \sim 14 \%$ at $\cos \theta=1$ and a deficit of $F \sim-2 \%$ at $\cos \theta=0$. An interesting feature is that the excess appears near the polar regions, which is opposite to the result obtained for $r_{\mathrm{e}} \geq 4.5 \mathrm{M}$, as considered in Section 3. Furthermore, the magnitude is much larger than the geometrical effect, which is estimated by extrapolating the empirical formula (13), and is $F \sim-6 \%$ at the largest deviation point $\cos \theta=1$. Thus, it is concluded that the number distribution strongly depends on other factors. As shown in Figure 5, the escape fraction for $r_{\mathrm{e}}=2.5 \mathrm{M}$ varies with a typical amplitude $\pm 10 \%$ around the average value. This effect overwhelms the modifications which occur during the propagation, although the excess at the polar regions is expected to be suppressed to a certain extent 
if the extrapolation still holds. The right panel of Figure [6] shows the results obtained for a smaller emission radius $r_{\mathrm{e}}=1.1 \mathrm{M}$. Note that the outer boundary of the ergoregion is given by $r=M(1+\sin \theta)$ in the extreme Kerr case, where the emission sphere is located within the ergoregion, except for the polar regions. The excess around the polar regions transforms into a great deficit of $-90 \%$, and the deviation from the average also becomes much larger. The origin of this feature can mainly be explained by the non-uniformity at the emission surface $r_{\mathrm{e}}$.

\section{Discussion}

A strong gravitational field affects the emission and motion of particles in several ways. For example, a central black hole plays an important role in forming the surrounding accretion disk, and hence determines the distribution of the particles which are emitted into phase space, i.e., the distributions of position and velocity. Strong gravitational fields also affect the propagation of particles. In this paper, we have concentrated on the latter in a Kerr space-time by calculating the trajectories of particles emitted from a 'spherical' shell. Our concern is not the orbit of a single particle, but the behavior of an assembly of particles. That is, the deviation from 'spherical symmetry' which occurs during the propagation of the particles which are initially uniformly distributed on the emission shell and are emitted in isotropic directions. We were able to extract the geometrical effect under an ideal situation for the emission at $r_{\mathrm{e}} \gtrsim 4.5 \mathrm{M}$. In this case, the particles are anti-collimated on average, i.e., they spread in a direction perpendicular to the rotation axis in Kerr space-time. This feature can be seen even in weak gravitational field with mass quadrupole moment, which partially accounts for the physical mechanism. The magnitude depends on the Kerr parameter and the emitting radius $r_{\mathrm{e}}$, and the deviation from uniform distribution amounts to a few percent in the case of $r_{\mathrm{e}}=4.5 \mathrm{M}$. With a further decrease in the radius, the geometrical effect increases, but the other effects, such as the absorption of particles in certain orbits, become important, and therefore our definition of collimation/anti-collimation becomes ambiguous for $r_{\mathrm{e}} \lesssim 4.5 M$.

This feature of anti-collimation along the rotation axis does not agree with the results of previous works at first sight. The main difference arises from the emission models used. Bičák et al. [4] derived orbital parameters of which a particle emitted in the radial direction is collimated toward the rotation axis. The condition satisfies $\mathcal{Q}<0$, which corresponds to the $\theta$ motion confined in the range $0<\theta<\pi / 2$ or $\pi / 2<\theta<\pi$ [11. We checked the condition in our numerical simulation, and found that the fraction of trajectories satisfying it is typically $\sim 0.1 \%$. The isotropic emission is assumed in our model, so that most trajectories are irrelevant to the collimation condition derived by Bičák et al. [4]. Williams [14] calculated the production and subsequent propagation of $e^{-} e^{+}$pairs in the Penrose process within the ergosphere by using a Monte Carlo simulation. The results show that escaping trajectories are collimated and that helical trajectories exist along the rotation axis. The initial emission within the ergosphere 
is highly constrained in the Penrose process [15], since one of the particles in the pair should be in a negative energy state. The emission model is therefore different from ours. Recently, Bini et al.[16] considered the outflow along the rotation axis, and showed that an initially spherical assembly of particles becomes elongated along the rotation axis during the propagation of the particles. In their model, the initial direction of the velocity is fixed along the rotation axis, which is again different from our model.

It is not easy to summarize the effects of a strong gravitational field on the collimation of emitted particles in a Kerr space-time while taking into account the results obtained in other works. In our model, the effect which occurs during the propagation has been clarified as a result of simplifying the emission model. We found the unanticipated result, i.e., no collimation along the spin axis, but rather spread toward equator. This anti-collimation effect along the rotation axis is mainly explained by mass quadrupole moment of the Kerr space-time. The geometrical modification integrated from the emission point to infinity is $\sim(a / M)^{2}\left(6 M / r_{\mathrm{e}}\right)^{2.5}$, which is not so large. We conclude that in order for the collimated outflow to form, the emission model should provide a much larger deviation in the emitting direction and in the spatial distribution, where the Kerr space-time has a considerable effect.

\section{Acknowledgments}

Numerical computation in this work was carried out using the PC Cluster System of the

Information Media Center in Hiroshima University. This work was supported in part by a Grant-in-Aid for Scientific Research from the Japan Society for the Promotion of Science (JSPS) (KT).

\section{References}

[1] McKinney, J. C., 2006, MNRAS, 368, 1561

[2] de Felice, F. and Curir, A., 1992, CQG, 9, 1303

[3] Semerák, O., 1995, Czech. J. Phys., 45, 1

[4] Bičák, J., Semerák, O. and Hadrava, P., 1993, MNRAS, 263, 545

[5] de Felice, F. and Carlotto, L., 1997, ApJ, 481, 116

[6] Karas, V. and Dovciak, M., 1997, Gen. Rel. Grav., 29, 1011

[7] de Felice, F. and Zanotti, O., 2000, Gen. Rel. Grav., 32, 1449

[8] Semerák, O., 1999, MNRAS, 308, 863

[9] Gariel, J., MacCallum, A. H., Marcilhacy, G. and Santos, N. O., 2007, gr-qc/0702123

[10] Misner, C. W., Thorne, K. S. and Wheeler, J. A., 1973, Gravitation(New York: Freeman)

[11] Chandrasekhar, S., 1983, The Mathematical Theory of Black Holes(Oxford: Oxford University Press)

[12] Frolov, P. V. and Novikov, D. I., 1998, Black Hole Physics(Dordrecht: Kluwer)

[13] Thorne, K. S., 1980, Rev. Mod. Phys., 52, 299

[14] Williams, R. K., 2004, ApJ, 611, 952

[15] Bardeen, J. M., Press, W. H. and Teukosky, S. A., 1972, ApJ, 178, 347

[16] Bini, D., de Felice, F. and Geralico, A., 2007, Phys. Rev., D76, 047502 